\title{
E-health Project Examination: Introduction of an Applicable Pulse Oximeter
}

\author{
Mona Nasseri \& Seyedeh Fatemeh Khatami Firoozabadi \\ Electrical Department, Central Tehran Branch, Islamic Azad University, Tehran, Iran \\ E-mail:nasseri.mona@hotmail.com, fateme.khatami@gmail.com
}

\begin{abstract}
In this paper, E-health is investigated due to its advantages such as reducing health care cost, saving time, and decreasing unnecessary going to a clinic or hospital. In this research oxygen saturation is chosen as a sample signal for E-health investigation, therefore an applicable method is introduced to calculate oxygen saturation and a package of pulse oximetry is designed to receive signal which is recorded and sampled by sound card of computer, by means of MATAB. The recorded data is sent to a doctor through internet, which can be demonstrated in spectra, prepared for further analysis and also a model for counting heart beats is proposed. The numerical results, which were gained in number of experiments by attending different volunteers, show its reliability. Proposed device of pulse oximetry is simple, portable, and just need a cable for connecting to a computer to send the data through internet for expert analysis.
\end{abstract}

Keywords: E-health, Oxygen saturation, Pulse oximetry

\section{Introduction}

This is a long time that technology has been used for improving health condition and facilitating medical care. Additionally, wireless systems create the ability of measuring vital parameters wherever (Mendelson, Y., et al, 2006; Moron, M. J., Casilari, E. et al, 2005). Telemedicine and telecare projects were proposed in last decades (Bashshur, R. L. et al., 1975; Bashshur, R., 1977) and all remote care services, using technologies such as internet to improve the health status of patients were called E-health (Marconi, J., 2002), which contains significant advantages such as quality enhancement of selfcare systems, manipulating of information technology and monitoring patient health condition not only at house but also in work place. This kind of system decreases the hospital admission which can be time consuming.

As mentioned before, useful facility which is a part of this technology is internet, enabling doctor to visit the patient virtually so that all vital signals are transferred through internet. One of the best signal recording methods is through computer sound card which provides doctor to convert the sound signal to spectra and analyze it. The computer software which is used to record and convert the sound in an appropriate frequency is MATLAB, saving the data in a matrix, making it easy to send through internet, then this matrix is demonstrated as a wave shape, using MATLAB.

One of the most important vital parameters is blood oxygen saturation, which is considered in this paper. Pulse oximeter is a tool to measure blood oxygen continuously. The first device was made by a German researcher in 1930s (Severinghaus, JW., 1986), which measured the oxygen saturation by transilluminating the colored light. In 1970s, the Hewlett-Packard company made a device which used light in more than 2 wavelengths (Mendelson, Y., 1992).

Today the accepted technology for pulse oximetry is none invasive which is based on Beer-Lambert law (Webster, J. G., 1997) and depicts a relation between oxygen and the amount of absorbed light by blood.

Pulse oximetry depends on the property of light absorption by hemoglobin, 2 molecule, oxidized hemoglobin $\left(\mathrm{HbO}_{2}\right)$ and reduced hemoglobin $(\mathrm{Hb})$, by different optical spectra in the wavelength range from $500 \mathrm{~nm}$ to $1000 \mathrm{~nm}$, which contains the red and infrared wavelengths (Webster, J. G., 1997; Moyle, J. T., 2002). Oxygen saturation percentage is denoted by $\mathrm{SaO}_{2}$ and a normal patient, have a saturation level between $96-99 \%$.

\section{The Design of pulse oximetry instrumentation}

As mentioned before red and infrared (IR) are used because of the characteristic of absorption of infrared light by oxidized hemoglobin and absorption of red light by reduced hemoglobin in different wavelengths (Mendelson, Y., 1992). In Pulse oximeter, there are two emitting LED in R and IR wavelengths, located on the nail and a photo diode on the other side of the finger which detects the light, passing through the finger.

\subsection{Transmitter circuit}

Two Red and IR LEDs, which are used in transmitter circuit in pulse oximeter, must not light up simultaneously. 
Therefore the pulse source is designed, using IC 4093 which produces two reverse signals as outputs that can turn on LEDs sequentially. The generated signals are designed in $1 \mathrm{KHz}$ frequency, due to the need of a signal by frequency higher than beat rate frequency. One output of transmitter circuit is used to turn on a LED and the other one, for switching on the second one. Figure 1, shows the transmitter circuit.

\subsection{Receiver circuit}

The light which passes through finger must be detected accurately, hence elimination of noises such as environmental light should be considered as an essential issue in designing of receiver circuit. To achieve this purpose, an appropriate photodiode should be chosen. By examination of diverse photodiode models, the 34B is used in the receiver circuit due to the desirable characteristics. This model receives the light in the range from ultra violet (UV) to infrared.

In the next step the variation of photodiode output current must be amplified by means of trans impedance OPAMP. Due to the existence of high noise, an ultra low noise amplifier should be used. The OP07 is chosen in this paper because of its availability and its low cost. Moreover photodiode includes a large resistance which its impact can be reduced by using parallel resistance locating in p-junction. A very large feedback resistance should be used for reinforcing the received voltage, which is shown in Figure 2.

The transmitter and receiver diodes are locating in a package which can cover finger and also isolates it of environmental light.

\subsection{Results of experiments}

One way to test the function of transmitter and receiver is evaluation of its operation versus the thickness of material that is located between them. Therefore different number of papers is put in device which shows the decrease of received voltage while the number of papers increases, the result is shown in Figure 3 that proves the precision of device.

In another experiment, the finger is put between transmitter and receiver and the output is recorded by sound card of computer in MATLAB program by "wavrecord" command, then data is analyzed in MATLAB in which peaks can be detected. Through peak detection, counting the heart beat would be possible. Simulink environment is chosen to detect and count peaks of output data, which are recorded by sound card.

Figure 4 shows samples of output in MATLAB with sampling rate of $26 \mathrm{~Hz}$, for 3 different subjects. Obviously the amplitude and starting point of each pulse is differed from one subject to another. The variations among subjects are natural due to diversity of oxygen density. The peak detection model is shown in Figure 5. The output data is sent to a Notch filter to suppress $50 \mathrm{~Hz}$ power line interference. Then by using maximum finder block, the number of heart beats is counted, according to adjusted threshold.

To evaluate the accuracy of device, the comparison between number of heart beats that was gained in experimental way (to feel person's pulse) and the results, which achieved by using designed device in 14 experiments, is shown in Table 1, which proves its acceptability.

Also some parameters like Detection and Detection Error Rate is used to demonstrate its exactitude.

$$
\begin{gathered}
\text { Detection }=\frac{\left(S_{N}-F_{P}-F_{N}\right)}{S_{N}} \\
\text { Detection Error Rate }=\frac{\left(F_{P}+F_{N}\right)}{S_{N}}
\end{gathered}
$$

Where,

$\mathrm{S}_{\mathrm{N}}$ : the whole samples in an experiment

$\mathrm{F}_{\mathrm{p}}$ : the number of samples which are determined heart beat by mistake

$\mathrm{F}_{\mathrm{N}}$ : the number of heart beats which are not determined

According to acquired results in 14 experiments, investigating Table 1, in the best case the Detection of $100 \%$ was achieved which lead to Detection Error Rate of $0 \%$, and in the worst case the Detection was $91.6 \%$ with Detection Error Rate of $8.3 \%$, which is for fifth experiment.

\section{Conclusion}

In this paper the E-health project was considered due to its impacts on expanding of new remote medical 
methods, which makes easier to take care of health condition of patients. For this research, oxygen saturation was chosen to investigate. Pulse oximetry is a method to determine this important vital signal, therefore a simple and applicable device was designed and produced which was used in different experiments. Data was recorded by sound card of computer and analyzed in MATLAB, which is able to count the number of heart beats by using Simulink environment, and the numerical results show its validity, additionally the data can be sent through internet for more expert analysis by physicians, which lead to decrease cost and save time.

For future work the combination of several devices such as pulse oximetry, ECG, body temperature measurement, according to different applications, seems to be essential for expanding E-health technology.

\section{References}

Bashshur, R. L., Armstrong, P. A., \& Youssef, Z. I. (1975). Telemedicine: Explorations in the use of telecommunications in health care. Springfield, IL: Charles C. Thomas. S

Bashshur, R., \& Lovett, J. (1977). Assessment of telemedicine: Results of the initial experience._Aviation Space and Environmental Medicine, 48(1): 65-70.

Marconi, J. (2002). E-Health: Navigating the Internet for Health Information Healthcare. Advocacy White Paper. Healthcare Information and Management Systems Society.

Mendelson, Y. (1992). Pulse oximetry: Theory and applications for noninvasive monitoring. Clinical Chemistry. 38(9):1601-1607.

Mendelson, Y., Duckworth, R. J., \& Comtois, G. (2006). A Wearable Reflectance Pulse Oximeter for Remote Physiological Monitoring. Proceedings of the 28th IEEE EMBS Annual International Conference New York City.

Moyle, J. T. (2002). Pulse Oximetry.( $2^{\text {nd }}$ ed.), London, BMJ Books.

Moron, M. J., Casilari, E., Luque, R., \& Gazquez, J. A. (2005). A Wireless Monitoring System for Pulse-oximetry Sensors. Proceedings of the 2005 Systems Communications, IEEE(ICW'05).

Severinghaus, JW., \& Astrup, PB. (1986). History of blood gas analysis. VI. Oximetry. Clin Monit, 2(4):270-88.

Webster, J. G. (1997). Design of Pulse Oximeters. (1 ed.) Taylor \& Francis ublishing.(chapter 4, 5).

Table 1. The comparison between number of heart beat in experimental way and using device

\begin{tabular}{|c|c|c|}
\hline $\begin{array}{c}\text { Experiment } \\
\text { number }\end{array}$ & $\begin{array}{c}\text { Experimental } \\
\text { number of heart } \\
\text { beats }\end{array}$ & $\begin{array}{c}\text { Counting heart } \\
\text { beats using } \\
\text { spectra }\end{array}$ \\
\hline 1 & 7 & 7 \\
\hline 2 & 8 & 7 \\
\hline 3 & 9 & 9 \\
\hline 4 & 10 & 10 \\
\hline 5 & 13 & 12 \\
\hline 6 & 14 & 14 \\
\hline 7 & 18 & 18 \\
\hline 8 & 21 & 22 \\
\hline 9 & 24 & 23 \\
\hline 10 & 30 & 28 \\
\hline 11 & 32 & 34 \\
\hline 12 & 35 & 36 \\
\hline 13 & 37 & 37 \\
\hline 14 & 70 & 68 \\
\hline
\end{tabular}




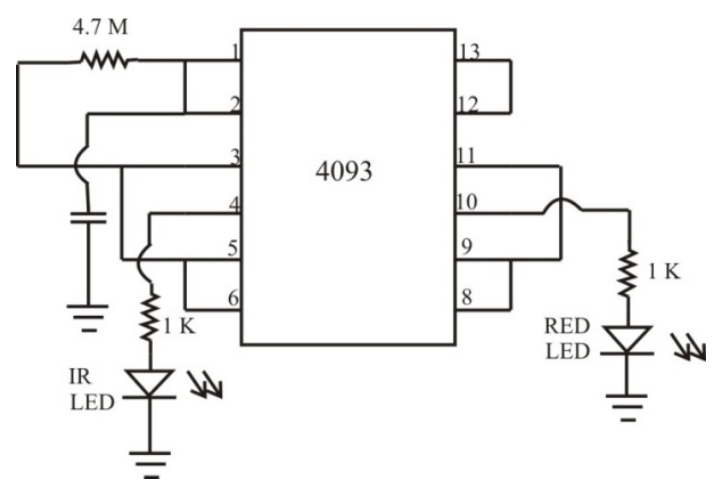

Figure 1. Transmitter circuit

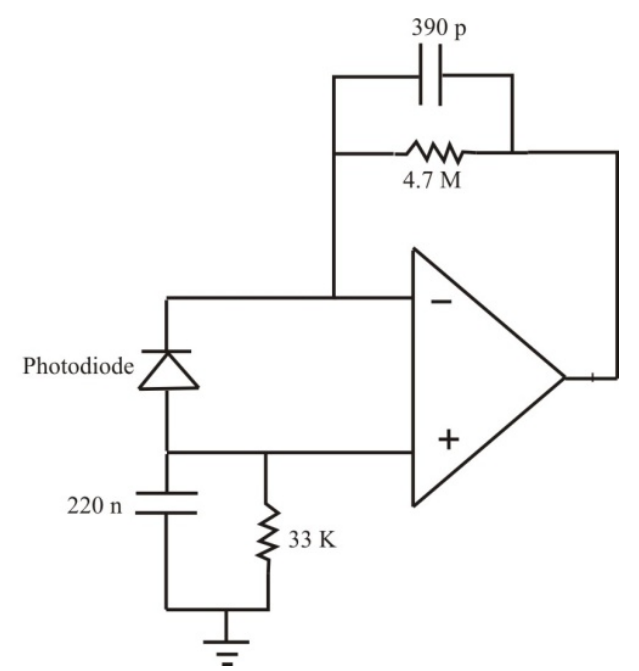

Figure 2. The designed circuit for amplifying the received signal

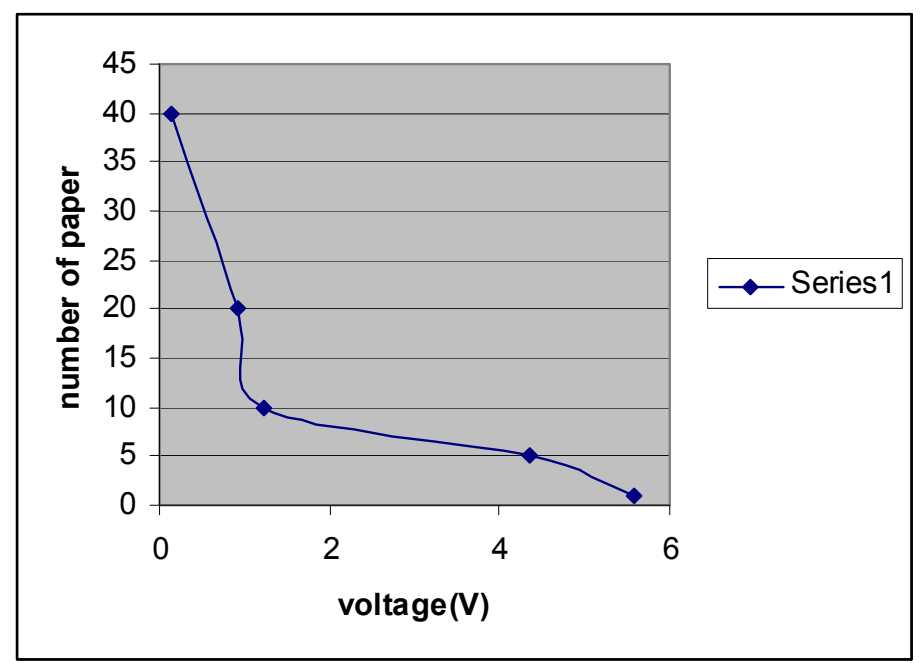

Figure 3. The variation of received voltage versus changing the number of papers 


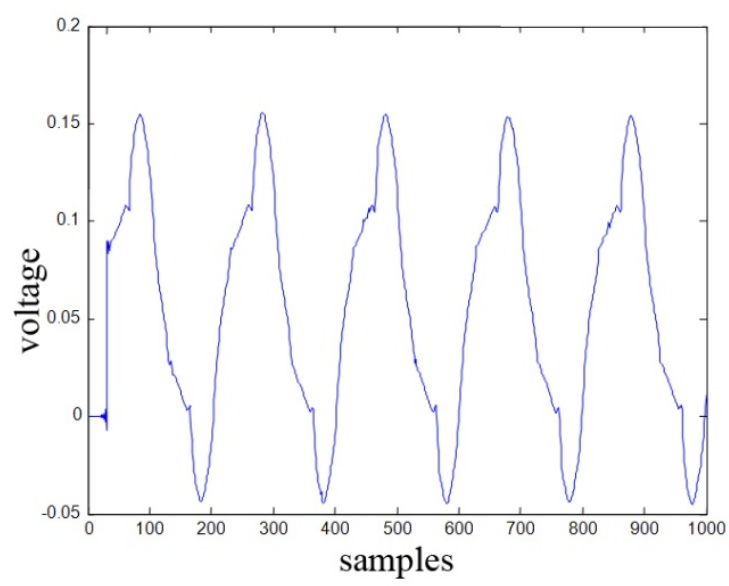

(a)

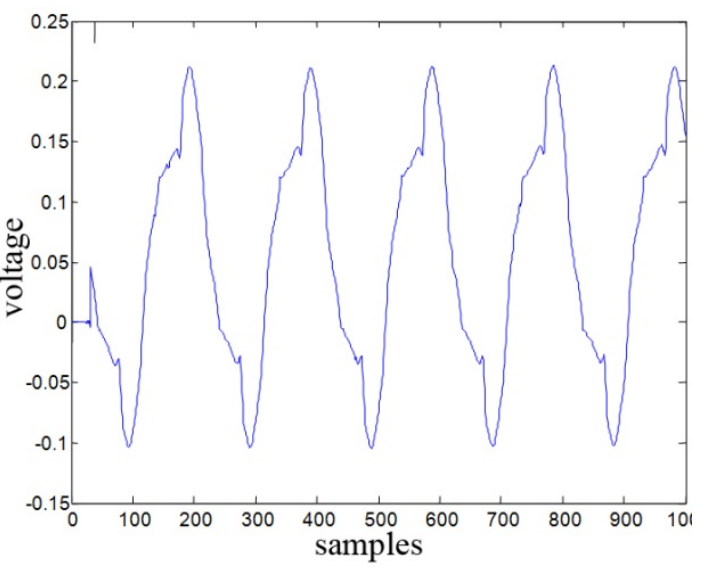

(b)

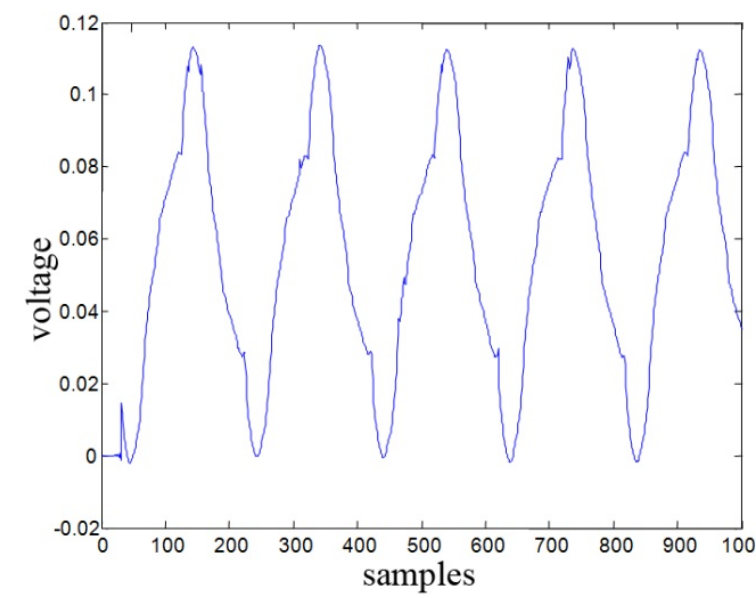

(c)

Figure 4. Output of pulse oximeter, depicting in MATLAB for three different volunteer

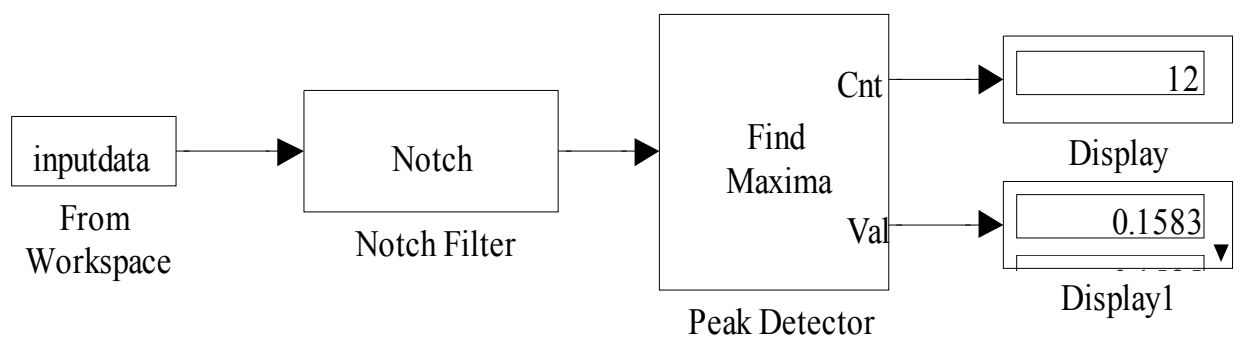

Figure 5. Peak detection model in MATLAB 